\title{
BMJ Open Association between lymphocyte-to- monocyte ratio (LMR) and the mortality of HBV-related liver cirrhosis: a retrospective cohort study
}

\author{
Jie Zhang, ${ }^{1}$ Guofang Feng, ${ }^{2}$ Ying Zhao, ${ }^{1}$ Juanwen Zhang, ${ }^{1}$ Limin Feng, ${ }^{1}$ \\ Jing Yang ${ }^{3}$
}

To cite: Zhang J, Feng G, Zhao $Y$, et al. Association between lymphocyte-tomonocyte ratio (LMR) and the mortality of HBV-related liver cirrhosis: a retrospective cohort study. BMJ Open 2015;5:e008033. doi:10.1136/bmjopen-2015008033

- Prepublication history for this paper is available online. To view these files please visit the journal online (http://dx.doi.org/10.1136/ bmjopen-2015-008033).

Received 25 February 2015 Revised 24 July 2015 Accepted 30 July 2015

CrossMark

1Department of Laboratory
Medicine, The First Affiliated
Hospital, College of Medicine,
Zhejiang University,
Hangzhou, China
2Department of Reproductive
Endocrinology, Women's
Hospital, School of Medicine,
Zhejiang University,
Hangzhou, China
${ }^{3}$ Department of Clinical
Laboratory, The Hospital of
Stomatology, Sun Yat-sen
University, Guangzhou, China

Correspondence to

Limin Feng;

flm@zju.edu.cn

\begin{abstract}
Objective: Infection with hepatitis B virus (HBV) remains a major cause of liver cirrhosis (LC) in China. Recent reports suggest that the lymphocyte-tomonocyte ratio (LMR) is a potential biomarker for predicting clinical outcomes. In our study, we investigated if LMR can be used as a prognostic marker of mortality in patients with HBV-related LC.

Design: A retrospective cohort study.

Setting: HBV-infected patients with LC and patients with chronic hepatitis $B$ infection (CHB) from the Department of Infectious Disease were enrolled and 240 healthy individuals were recruited from the healthcare centre at the First Affiliated Hospital of Zhejiang University.
\end{abstract}

Participants: 479 HBV-infected patients with LC, 134 patients with $\mathrm{CHB}$ and 240 healthy individuals were enrolled.

Primary and secondary outcome measures: The receiver operating characteristic (ROC) curve and multivariable logistic regression analysis after adjusting for total protein, albumin, total bilirubin and the model for end-stage liver disease (MELD) score were used to evaluate the power of LMR for predicting 1 year mortality in patients with LC.

Results: The LMR was statistically lower in patients with LC. The MELD score and mortality were statistically higher in patients with LC compared with the CHB and control groups. The area under the ROC curve, cut-off values, sensitivity and specificity of LMR for predicting mortality LC in the training cohort were 0.817 (95\% Cl 0.746 to $0.888 ; p<0.001), 2.10,82.6$ and $78.8 \%$, and these data were confirmed in the validation cohort. The multivariate logistic regression analysis showed that LMR was an independent predictive factor of mortality in LC (OR $2.370,95 \% \mathrm{Cl}$ (1.070 to 5.249); $p=0.033$ ).

Conclusions: Our results strongly suggest that low LMR can be considered as an independent biomarker for predicting mortality in patients with LC.

\section{INTRODUCTION}

Liver cirrhosis (LC) is a common hepatic disease in China that represents an increasing

\section{Strengths and limitations of this study}

- Lymphocyte-to-monocyte ratio (LMR) was lower in the liver cirrhosis (LC) group, especially in the non-surviving group, compared with the control group and the chronic hepatitis B infection group.

- LMR was closely correlated to the model for end-stage liver disease (MELD) score.

- LMR was an easy parameter to achieve and the power for predicting mortality of LMR was similar to that of MELD.

- Low LMR levels were independent factors for predicting mortality in patients with LC.

- This was a retrospective study.

cause of morbidity and mortality. ${ }^{1}{ }^{2}$ Hepatitis $B$ virus (HBV) infection remains a major cause of LC in China, with a $3 \%$ yearly incidence of decompensated cirrhosis. ${ }^{3}$ Systemic inflammatory response syndrome (SIRS) is relatively common in patients with complicated cirrhosis. ${ }^{4}{ }^{5}$ It can further deteriorate liver function, maximise the risk of complications and increase the mortality rate of patients with LC. ${ }^{4}$ SIRS is usually measured by peripheral blood count-based parameters, such as neutrophils, lymphocytes, monocytes, red blood cell distribution width (RDW), mean platelet volume or platelet count. These parameters have been reported to be independent predictive markers of clinical outcome in cancer and different states of HBV-related hepatic disorders. ${ }^{6-10}$ Among these inflammatory parameters, the neutrophil-lymphocyte ratio (NLR), RDW and monocyte ratio have been proposed as easily accessible and reliable markers. ${ }^{6-8} 11$ Several recent studies suggest that the lymphocyte-to-monocyte ratio (LMR) is a cheap, readily available and reproducible test with potential for predicting clinical 
outcomes of patients with solid tumours and haematological malignancy, including nasopharyngeal carcinoma, colorectal cancer, pancreatic cancer and lymphoma. ${ }^{12-15}$ Moreover, Merekoulias et $a l^{16}$ found that in $90 \%$ of patients who had influenza virus, lymphopenia and/or monocytosis, LMR could be used as a time-saving and cost-effective screening test for influenza virus infection, leading to early antiviral treatment and a decreased incidence of complications. Assuming that there may be an association between LMR and LC severity, we investigated the potential prognostic value of LMR as a biomarker in HBV-related LC.

To the best of our knowledge, there are no data on LMR as a LC diagnostic measure. We therefore performed a retrospective cohort study to investigate the association between LMR in peripheral blood in patients with LC, with special emphasis on the value of LMR for predicting the mortality of patients with LC.

\section{SUBJECTS AND METHODS}

\section{Subjects}

We continuously analysed all 547 patients with HBV-related LC from the Department of Infectious Disease, The First Affiliated Hospital, School of Medicine, Zhejiang University, between October 2012 and October 2013. 68 patients with LC with a concurrent infection of hepatitis $\mathrm{C} / \mathrm{D} / \mathrm{E} / \mathrm{G}$ virus $(\mathrm{n}=3)$, HIV $(\mathrm{n}=1)$, hepatocellular carcinoma (HCC, $\mathrm{n}=56$ ), alcoholic cirrhosis $(n=5)$, schistosomiasis cirrhosis $(n=1)$ and any autoimmune liver disease $(n=2)$ were excluded. The remaining 479 patients with LC were enrolled in our retrospective cohort study. All clinical data were retrieved from medical records at the Department of Infectious Disease. 134 patients with chronic hepatitis B infection $(\mathrm{CHB})$, with no statistical differences in age and gender versus patients with LC, were selected from the Department of Infectious Disease without a concurrent infection of hepatitis $\mathrm{C} / \mathrm{D} / \mathrm{G}$ virus, HIV, HCC and any autoimmune liver disease between October 2012 and October 2013. CHB and LC were diagnosed according to the criteria of the $2000 \mathrm{Xi}$ 'an viral hepatitis management scheme. ${ }^{17}$ LC was diagnosed on the basis of the history of liver disease, clinical manifestations, laboratory tests, imaging tests and, whenever feasible, liver biopsy. ${ }^{17}$ Decompensated cirrhosis was defined by the presence of jaundice, ascites, variceal haemorrhage or hepatic encephalopathy. ${ }^{17} 18$ The causes of admission in patients with LC without decompensation were mainly jaundice, hypodynamia and portal hypertension manifestations (oesophageal varices, hypersplenism). The causes of admission in patients with LC with decompensation were ascites, upper gastrointestinal bleeding (oesophageal varices), hepatic encephalopathy, hepatorenal syndrome and infection. CHB was defined as hepatitis B or hepatitis B surface antigen ( $\mathrm{HBsAg}$ ) positivity for $>6$ months, and persistently positive HBsAg and/or HBV DNA. ${ }^{17} 18$ The LG group was subdivided into two subgroups according to mortality at 1-year follow-up. 227 patients with LC and 33 with CHB were under antiviral therapy before admission, 189 patients with LC and 76 with CHB were under antiviral therapy after admission, altogether 416 patients with LC $(86.8 \%)$ and 109 $(81.3 \%)$ with $\mathrm{CHB}$ were under antiviral therapy. For patients with LC and CHB discharged from hospital, 1 year prognostic information was obtained by checking medical records or by contacting the patients' family members. 108 patients with LC were decompensated. 92 LC patients died from the following causes: upper gastrointestinal bleeding $(\mathrm{n}=40)$; hepatic encephalopathy $(\mathrm{n}=28)$; hepatorenal syndrome $(\mathrm{n}=15)$; infection $(n=5)$; electrolyte disturbance $(n=2)$; multiple organ failure $(n=1)$; and respiratory failure $(n=1) .240$ healthy controls with no statistical differences in age and gender versus patients with $\mathrm{LC}$ were selected from a health examination population which underwent a general health check-up that included a physical examination and some clinical laboratory tests at the Health Care Centre of the First Affiliated Hospital of Medical College of Zhejiang University between September 2013 and October 2013. They corresponded to HBsAg-negative individuals with normal liver function, normal renal function and no infection. 134 patients with $\mathrm{CHB}$ and 240 healthy controls were used to compare basic characteristics with 479 patients with LC.

\section{Laboratory assessment}

All venous blood samples were obtained in the morning following a $12 \mathrm{~h}$ fast, within $24 \mathrm{~h}$ after admission. All study participants were subjected to the following determinations: serum total protein (TP), albumin (ALB), total bilirubin (TB), alanine aminotransferase (ALT), aspartate aminotransferase (AST), triglyceride (TG), total cholesterol $(\mathrm{Tch})$, creatinine $(\mathrm{Cr})$, prothrombin time (PT), complete blood cell counts, LMR in peripheral blood, international normalised ratio (INR) and the model for end-stage liver disease (MELD) score based on TB, Cr, INR and PT. ${ }^{18}$ Complete blood cell counts were determined using a Sysmex XE-2100 automated haematology analyser (Sysmex Corp, Kobe, Japan) with Sysmex reagents.

\section{Statistical analysis}

Statistical analysis was performed using SPSS V.16.0 (SPSS Inc, Illinois, USA). Data were presented as mean $\pm \mathrm{SD}$, median (range) or categorical data as percentages, if appropriate. The differences between the two groups were assessed with an independent sample $\mathrm{t}$ test, the Mann-Whitney $\mathrm{U}$ test or the $\chi^{2}$ test, if appropriate. Multiple comparisons were performed by one-way analysis of variance or Kruskal-Wallis $\mathrm{H}$ tests, if appropriate. The LC cohorts were randomly divided into estimation and validation cohorts by random number generators. Spearman's correlation test was used in correlation analyses. The receiver operating characteristic (ROC) curve and cut-off values of LMR were obtained, and area under 
ROC (AUROC) curve was calculated to identify the best LMR and/or the MELD score for predicting mortality in patients with LC. For the AUROC analysis of the combined 1/LMR and MELD score for predicting mortality in patients with LC, predictive models of 1/LMR, MELD and $1 /$ LMR+MELD were first developed by binary logistic regression analyses, respectively. Probabilities of 1/LMR, MELD and 1/LMR+MELD were then generated, respectively, and used as three new input variables for the ROC curve analysis (shown in figure 2). These parameters were selected by stepwise regression, and multivariate logistic regression analyses were used to evaluate if low LMR was an independent factor for predicting mortality in patients with LC by an unadjusted model and adjusting for TP, ALB, TB and the MELD score. The high LMR group was used as the reference category. Statistical significance was defined at $\mathrm{p}<0.05$.

\section{RESULTS}

\section{Patient characteristics}

There were 479 patients with LC, 134 patients with CHB and 240 healthy controls enrolled in our study. The patient characteristics are listed in table 1 . No statistical differences were observed for gender and age between the three groups, whereas TP, ALB, TB, ALT, AST, TG, Tch, Cr, INR, LMR and white cell count (WCG) count had statistical differences (all $\mathrm{p}<0.05$ ). The MELD score and mortality of the LC group were statistically higher than those of the CHB group $(\mathrm{p}<0.001)$.
LMR is lower in LC, especially in the non-surviving group The LMR was significantly lower in the LC group compared with the control group (2.77 vs 5.30, respectively) and the CHB group (2.77 vs $3.64 ; \mathrm{p}<0.01)$. The clinical characteristics and differences in variables between non-surviving and surviving patients with LC are presented in table 2. The non-surviving patients had a lower LMR (figure 1), TP, ALB and Tch, and a higher MELD score, TB, ALT, AST, TG, Cr, INR, WCC, monocytes and rate of decompensated cirrhosis, compared with surviving patients. The median and range of lymphocyte count of the non-surviving group were slightly lower than those of the surviving group, but the difference did not reach statistical significance. These data indicate that the lower LMR in the non-surviving group was mainly due to an increased number of monocytes and secondarily due to decreased lymphocytes. LMR resulted in no significant differences in patients with LC whose primary cause of death was upper gastrointestinal bleeding, hepatic encephalopathy or hepatorenal syndrome $(1.35(0.35-17.75), 1.42$ $(0.27-18.20), 1.39(0.39-18.25), \mathrm{p}=0.955)$.

\section{LMR is correlated to the MELD score}

The LMR in the LC group negatively correlated with the MELD score $(\mathrm{r}=-0.241 ; \mathrm{p}<0.05)$, especially in nonsurviving patients with LC; LMR negatively correlated with the MELD score with a higher correlation coefficient $(r=-0.354 ; p=0.013$ ) compared with LMR in surviving patients with LC.

Table 1 Basic characteristics of enrolled participants

\begin{tabular}{|c|c|c|c|c|}
\hline Variables & Control (240) & CHB (134) & LC (479) & p Value \\
\hline Female/male & $61 / 179$ & $34 / 100$ & $126 / 353$ & 0.956 \\
\hline Age (year) & $50.6 \pm 9.69$ & $48.9 \pm 8.04$ & $50.8 \pm 10.8$ & 0.163 \\
\hline HBsAg-positive (yes/no) & $0 / 240$ & $134 / 0$ & $479 / 0$ & - \\
\hline HBeAg-positive (yes/no) & $0 / 240$ & $66 / 68$ & $184 / 295$ & $0.024^{*}$ \\
\hline $\mathrm{TP}(\mathrm{g} / \mathrm{L})$ & $71.6 \pm 3.79$ & $67.3 \pm 6.83 \dagger$ & $62.9 \pm 8.48 \dagger^{\star}$ & $<0.001$ \\
\hline ALB (g/L) & $46.2 \pm 3.17$ & $37.4 \pm 5.95 \dagger$ & $33.2 \pm 5.61 \dagger^{\star}$ & $<0.001$ \\
\hline TBIL $(\mu \mathrm{mol} / \mathrm{L})$ & $12(6-49)$ & $21.5(5-309) \dagger$ & $31(5-839) \dagger^{*}$ & $<0.001$ \\
\hline ALT (Ü/L) & $17(7-48)$ & $61(9-1838) \dagger$ & $29(4-1882) \dagger^{*}$ & $<0.001$ \\
\hline AST (U/L) & $19(12-46)$ & $48(16-1235) \dagger$ & $40(8-4094) \dagger^{*}$ & $<0.001$ \\
\hline TG (mmol/L) & $1.08(0.41-1.70)$ & $1.33(0.44-4.14) \dagger$ & $0.79(0.3-3.59) \dagger^{*}$ & $<0.001$ \\
\hline Tch (mmol/L) & $4.66(2.40-5.86)$ & $4.04(1.6-8.17) \dagger$ & $2.89(0.74-9.73) \dagger$ & $<0.001$ \\
\hline $\mathrm{Cr}(\mu \mathrm{mol} / \mathrm{L})$ & $73(39-100)$ & $65(29-154) \dagger$ & $66(30-729) \dagger$ & 0.002 \\
\hline INR & $0.94 \pm 0.05$ & $1.21 \pm 0.23 \dagger$ & $1.55 \pm 0.78 \dagger^{*}$ & $<0.001$ \\
\hline WCC $\left(10^{12} / \mathrm{L}\right)$ & $5.6(4.0-9.4)$ & $4.75(2-12) \dagger$ & $3.9(0.8-32.8) \dagger^{*}$ & $<0.001$ \\
\hline LMR & $5.30(1.4-13.2)$ & $3.64(0.65-9.61) \dagger$ & $2.77(0.27-18.25) \dagger^{*}$ & $<0.001$ \\
\hline MELD score & - & 5.89 (0-23.63) & $9.89(0-57.17)$ & $<0.001^{*}$ \\
\hline Mortality (yes/no) & - & $1 / 133$ & $92 / 387$ & $<0.001^{*}$ \\
\hline
\end{tabular}

Data were presented as mean \pm SD and median (range).

$\mathrm{p}$ Value: comparison among these three groups.

*LC group versus the CHB group.

$t p$ Value $<0.05$ versus the control group.

ALB, albumin; ALT, alanine aminotransferase; AST, aspartate aminotransferase; CHB, chronic hepatitis B; Cr, creatinine; HBeAg, hepatitis B e antigen; HBsAg, hepatitis B surface antigen; INR, international normalised ratio; LC, liver cirrhosis; LMR, lymphocyte-to-monocyte ratio; MELD score, model for end-stage liver disease score; TB, total bilirubin; Tch, total cholesterol; TG, triglyceride; TP, total protein; WCC, white cell count. 
Table 2 The clinical characteristics and differences in variables between non-surviving and surviving patients with LC

\begin{tabular}{|c|c|c|c|}
\hline Variables & Non-surviving $(n=92)$ & Surviving ( $n=387)$ & p Value \\
\hline Female/male & $30 / 62$ & $96 / 291$ & 0.127 \\
\hline Age (year) & $53.8 \pm 10.3$ & $50.1 \pm 10.8$ & 0.003 \\
\hline $\mathrm{TP}(\mathrm{g} / \mathrm{L})$ & $56.4 \pm 8.40$ & $64.5 \pm 7.74$ & $<0.001$ \\
\hline ALB (g/L) & $29.7 \pm 5.17$ & $34.0 \pm 5.40$ & $<0.001$ \\
\hline TBIL ( $\mu \mathrm{mol} / \mathrm{L})$ & 292.5 (9-839) & $27(5-836)$ & $<0.001$ \\
\hline ALT (U/L) & $48(4-1882)$ & $27(5-475)$ & $<0.001$ \\
\hline AST (U/L) & $66(10-4094)$ & $37(8-440)$ & $<0.001$ \\
\hline $\mathrm{TG}(\mathrm{mmol} / \mathrm{L})$ & $0.88(0.30-2.15)$ & $0.76(0.33-3.59)$ & 0.022 \\
\hline Tch (mmol/L) & $1.83(0.74-5.29)$ & 3.02 (0.94-9.73) & $<0.001$ \\
\hline $\mathrm{Cr}(\mu \mathrm{mol} / \mathrm{L})$ & 73.5 (30-729) & $65(30-326)$ & $<0.001$ \\
\hline INR & $2.23 \pm 1.51$ & $1.39 \pm 0.28$ & $<0.001$ \\
\hline WCC $\left(10^{9} / \mathrm{L}\right)$ & $6.75(0.8-24.9)$ & $3.6(0.9-32.8)$ & $<0.001$ \\
\hline Monocytes ( $\left.10^{9} / \mathrm{L}\right)$ & $0.73(0.04-3.16)$ & $0.33(0.05-2.0)$ & $<0.001$ \\
\hline Lymphocyte $\left(10^{9} / \mathrm{L}\right)$ & $0.9(0.1-4.3)$ & $1.00(0.10-5.40)$ & 0.166 \\
\hline LMR & $1.41(0.27-18.25)$ & $3.10(0.38-14.58)$ & $<0.001$ \\
\hline MELD score & $22.94(0.84-57.17)$ & $8.49(0-35.33)$ & $<0.001$ \\
\hline Decompensated cirrhosis (yes/no) & $82 / 10$ & $26 / 361$ & $<0.001$ \\
\hline
\end{tabular}

\section{The power for predicting 1 year mortality of LMR}

The enrolled 479 patients with LG were randomly divided into two cohorts: the training cohort $(n=239)$ and the validation cohort $(\mathrm{n}=240)$. The ROC curve analyses of the training cohort were applied to estimate LMR and the MELD score to predict the mortality of patients with LC (figure 2). LMR was changed into $1 /$ LMR by inverse transformation. The AUROCs of $1 /$ LMR and the MELD score were 0.817 (95\% CI 0.746 to $0.888 ; \mathrm{p}<0.001$ ) and 0.868 (95\% CI 0.795 to 0.941 ; $\mathrm{p}<0.001$ ), respectively. The cut-off values, sensitivity and specificity of MELD were 19.1, 73.9 and 96.4\%. LMR values were $2.10,82.6$ and $78.8 \%$. When $1 / \mathrm{LMR}$ and the MELD score were combined, the AUC was $0.876(95 \%$ CI 0.808 to $0.945 ; \mathrm{p}<0.001$ ), only slightly higher than the AUC of the MELD score, and neither the specificity $(71.7 \%)$ nor the sensitivity $(96.9 \%)$ was significantly improved. Applying the LMR to the validation cohort, the AUROCs of 1/LMR, the MELD score and 1/LMR
+MELD were 0.773 (95\% CI 0.692 to $0.854 ; \mathrm{p}<0.001$ ), 0.887 (95\% CI 0.829 to $0.945 ; \mathrm{p}<0.001$ ), 0.890 (95\% CI 0.836 to $0.944 ; \mathrm{p}<0.001)$, respectively. There were no significant differences in the AUCs of LMR between the estimation and validation cohorts $(\mathrm{Z}=0.741, \mathrm{p}=0.053)$. To summarise, LMR was an easy parameter to achieve and the power for predicting mortality of LMR was similar to that of MELD.

\section{LMR is an independent prognostic factor of mortality} in multivariate analysis

MELD, low $\operatorname{LMR}(\mathrm{LMR} \leq 2.10$, with high $\mathrm{LMR}>2.10$ as a reference), TP, TB and ALB were selected by stepwise regression from the above parameters $(\mathrm{p}=0.025,<0.001$, $0.048,0.006,<0.001$ and 0.021 , respectively) with forward selection. Subsequent multivariate logistic regression analysis showed that low LMR was an independent factor for predicting mortality in patients with LC (table 3 ).
Figure 1 The box plots of the MELD score and LMR between surviving and non-surviving patients with LC. LMR, lymphocyte-to-monocyte ratio; MELD score, model for end-stage liver disease score.
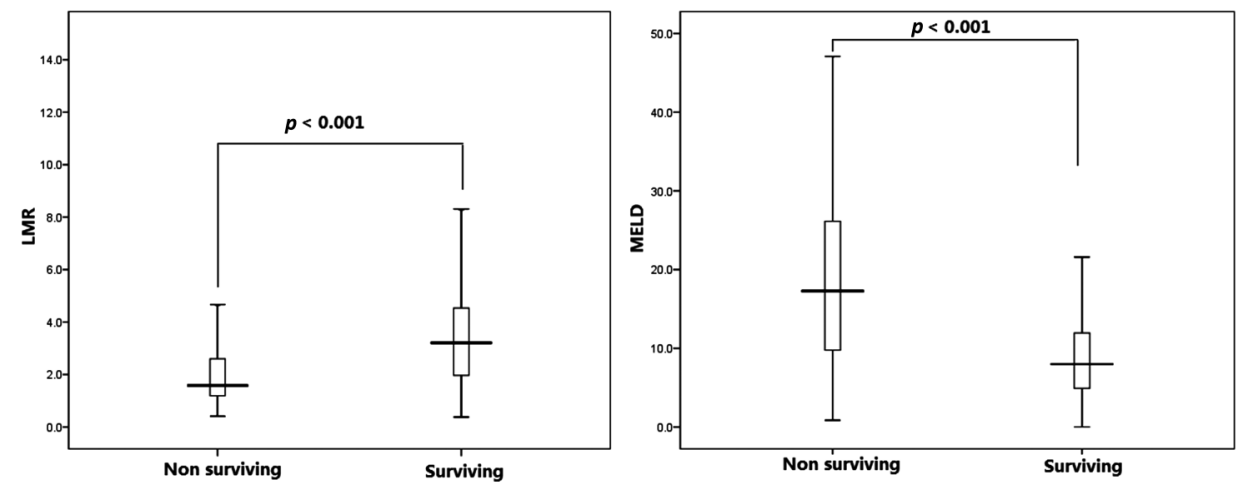


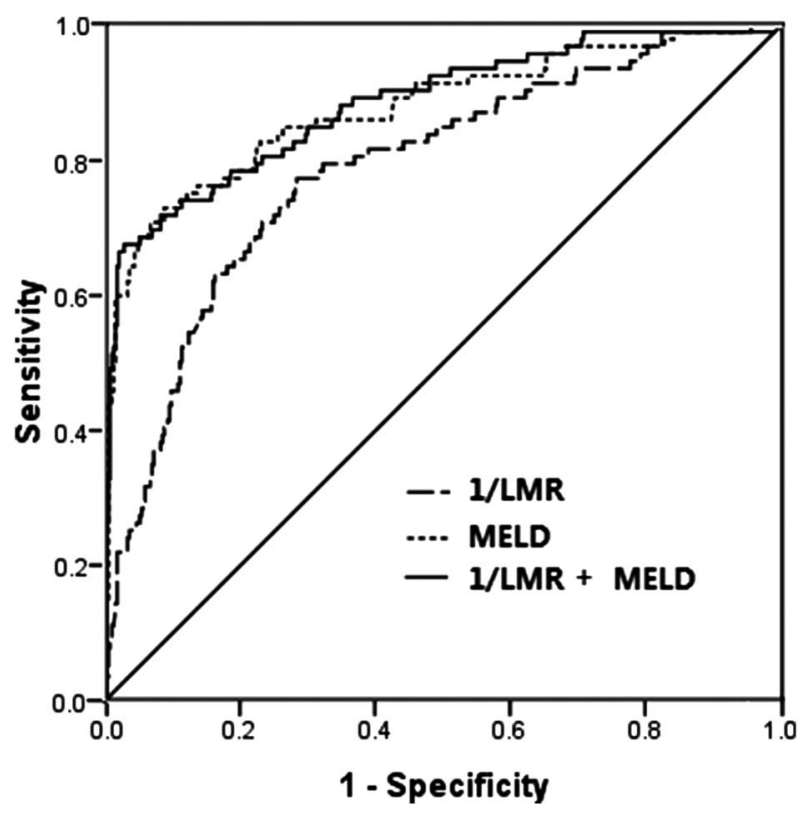

Figure 2 ROC curve analysis for predicting mortality by LMR and the MELD score in the training cohort. LMR, lymphocyte-to-monocyte ratio; MELD score, model for end-stage liver disease score; 1/LMR+MELD, 1/LMR combined with MELD; ROC, receiver operating characteristic.

\section{DISCUSSION}

In the present retrospective study of HBV-LC, a significant negative association was found between LMR in the peripheral blood and the MELD score. LMR of patients with LC was statistically lower, and the MELD score and mortality of patients with LC were statistically higher than those of the CHB and control groups, especially in the non-surviving LC subgroup. Moreover, low LMR was an independent predictive factor of mortality. These results provide the first evidence for an association between LMR and mortality in patients with LC.

Each year, approximately $2-5 \%$ of patients with compensated cirrhosis develop decompensation; patients with decompensated cirrhosis mainly die of cirrhosis-related complications, and the prognosis of decompensated cirrhosis is markedly worse, with a 5-year survival of $14-35 \%$ compared with $84 \%$ in compensated cirrhosis. ${ }^{19} 20$ Patients with decompensated cirrhosis also frequently

Table 3 ORs of low LMR for predicting mortality in patients with LC

\begin{tabular}{lll}
\hline Models & OR $(95 \% \mathbf{C l})$ & p Value \\
\hline Model 1 & $8.623(5.051$ to 14.721$)$ & $<0.001$ \\
Model 2 & $3.324(1.571$ to 7.035$)$ & $<0.001$ \\
Model 3 & $2.370(1.070$ to 5.249$)$ & 0.033 \\
\hline
\end{tabular}

ORs of low LMR were determined using high LMR as reference; model 1: unadjusted; model 2: adjusted for TP, ALB and TB; model 3: adjusted for TP, ALB, TB and the MELD score. LMR, lymphocyte-to-monocyte ratio; TP, total protein; TB, total bilirubin; ALB, albumin; MELD score, model for end-stage liver disease score. present with more than one facet of liver decompensation, and should then receive liver intense medical care and transplantation evaluation. ${ }^{19}$ In our non-surviving group, most patients had decompensated cirrhosis, and their LMR values were significantly lower than those of the surviving group where most patients had compensated cirrhosis. LMR was significantly correlated to the MELD score with a low $(r)$ correlation coefficient. However, the $r$ value in non-surviving patients with LC was higher than in surviving patients with LC, indicating that the LMR changes in non-surviving patients with LC were more pronounced, which coincided with table 3 results.

Bacterial infections are an important cause of morbidity and mortality in patients with LC due to an impaired immune function, together with an increased passage of bacteria from the gut (bacterial translocation). ${ }^{4}{ }_{5} \quad 21$ Once infection occurs, it may lead to SIRS, which can cause serious complications such as severe sepsis, renal dysfunction, encephalopathy, coagulopathy and multiple organ failure. ${ }^{21}$ SIRS occurs more frequently in patients with advanced cirrhosis and portal hypertension, and is associated with severity of liver disease and increased risk of death in patients with LC. ${ }^{45}$ The mortality of patients with LC with infection has been reported to be more than twice that of patients without infection. ${ }^{21}$ Monocytes are central mediators of the immune response and play a crucial role in the pathogenesis of LC. Endotoxin leads to monocyte activation and promotes the release of proinflammatory cytokines such as interleukin-1 (IL-1), IL-6, tumour necrosis factor $\alpha$ and interferon $\gamma$ into the serum. This release is proportional to liver disease severity. These cytokines act in an autocrine and paracrine fashion and result in the recruitment of inflammatory effector cells, such as polymorphonuclear cells. ${ }^{21-23}$ The subsequent activation of nitric oxide (NO) via the cytokine cascade leads to vasodilation. ${ }^{24}$ Endotoxin, cytokines and NO are key elements in the pathogenesis of circulatory abnormalities in $\mathrm{LC}$ with infection. $\mathrm{Li}$ and $\mathrm{Sun}^{25}$ found that monocytes in patients with HBV-related LC positively correlated with the endotoxin level and cirrhosis severity based on the Child-Pugh classification, indicating that the endotoxin-driven monocyte activation was an important factor of SIRS and multiple organ failure. Lee et $a l^{11}$ found that patients with LC with HCC had a high monocyte ratio and that a preoperative monocyte ratio $>7 \%$ was an independent risk factor for survival after hepatic resection. Immune paralysis, defined as decreased human leucocyte antigen-DR (HLA-DR) expression on monocytes and indicating immune dysfunction, was found in patients with LC. HLA-DR expression is a direct marker of monocyte function and a protective immune response in patients with LC. ${ }^{26}$ Monocyte HLA-DR expression is significantly reduced in those patients and falls in proportion to cirrhosis severity. $^{27} 28$ Therefore, patients with LC may have a high monocyte count but low monocyte HLA-DR expression for systemic inflammatory response and immune paralysis. Early diagnosis and treatment of infections can 
significantly reduce morbidity and improve survival of patients with LC. ${ }^{422} 242529$

Inflammatory stimuli mainly affect the numbers of monocytes in the peripheral blood in patients with LC, which contributes to LMR changes. In addition, the present study showed that lymphocytes in the nonsurvival group showed a trend towards lower levels as compared with the survival group, without reaching statistical significance. Such a decline might be attributed to lymphocytopenia. ${ }^{30} 31$ This is in accordance with Leithead et al, who found that a lower lymphocyte count was associated with mortality in patients with end-stage cirrhosis listed for liver transplantation. ${ }^{28}$ Lombardo $e t a l^{31}$ also found that the progressive and severity-related decrease in peripheral blood $\mathrm{T}$ lymphocyte suggested a progressive impairment of protective immune function in LC. Therefore, high monocytes together with low lymphocytes may reflect the severity and progression of liver injury in patients with LC.

LMR has been shown to be associated with tuberculosis and influenza virus infection. ${ }^{16}{ }^{32}$ Recently, LMR has also been reported to predict survival and prognosis in various patient populations with malignant diseases, ${ }^{12-15}$ and a decreased LMR has been shown to be significantly associated with a high risk for critical limb ischaemia in patients with peripheral arterial occlusive disease. ${ }^{33}$ Compared with another novel inflammation index, the ability of NLR to predict mortality (AUROC) in patients with $\mathrm{LC}^{34}$ was similar to that of LMR in our study. In our study, LMR was associated with the MELD score, the power for predicting mortality of LMR was similar to that of MELD, and was an independent predictive factor of mortality. In addition, LMR is an easily available and low price biomarker. However, it should be noted that this was a retrospective study so that prospective cohorts are warranted in order to confirm the present data. Another study limitation was that a 1:1 ratio was not adopted for setting up the control groups. Moreover, these findings may only apply to patients with HBV-related LC and therefore need to be validated in other aetiologies of LC by future prospective clinical trials.

Acknowledgements The authors thank Medjaden Bioscience Limited for helping in proofreading and editing the English of the manuscript.

Contributors LF designed the experiments. GF, JuZ and JZ performed the experiments. $Y Z$ and $J Y$ analysed and interpreted all the data. $J Z$ and $Y Z$ wrote the main manuscript text. All authors reviewed the manuscript.

Funding This work was supported by grants from the Department of Education Foundation of Zhejiang Province (Y201330146), the Science Foundation of the Health Bureau of Zhejiang Province (No. 2013KYB116) and the Natural Science Foundation of China of Zhejiang Province (LY15H190002).

Competing interests None declared.

Patient consent Obtained.

Ethics approval This study was approved by the Ethics Committee of the First Affiliated Hospital of the Medical College at Zhejiang University in China and was performed in accordance with the Helsinki Declaration.
Provenance and peer review Not commissioned; externally peer reviewed.

Data sharing statement No additional data are available.

Open Access This is an Open Access article distributed in accordance with the Creative Commons Attribution Non Commercial (CC BY-NC 4.0) license, which permits others to distribute, remix, adapt, build upon this work noncommercially, and license their derivative works on different terms, provided the original work is properly cited and the use is non-commercial. See: http:// creativecommons.org/licenses/by-nc/4.0/

\section{REFERENCES}

1. Wang X, Lin SX, Tao J, et al. Study of liver cirrhosis over ten consecutive years in Southern China. World $J$ Gastroenterol 2014;20:13546-55.

2. Tsochatzis EA, Bosch J, Burroughs AK. Liver cirrhosis. Lancet 2014;383:1749-61.

3. Wang SB, Wang JH, Chen J, et al. Natural history of liver cirrhosis in south China based on a large cohort study in one center: a follow-up study for up to 5 years in 920 patients. Chin Med J (Engl) 2012;125:2157-62.

4. Behroozian R, Bayazidchi M, Rasooli J. Systemic inflammatory response syndrome and MELD score in hospital outcome of patients with liver cirrhosis. Middle East J Dig Dis 2012;4:168-72.

5. Abdel-Khalek EE, El-Fakhry A, Helaly M, et al. Systemic inflammatory response syndrome in patients with liver cirrhosis. Arab $J$ Gastroenterol 2011;12:173-7.

6. Karagoz E, Ulcay A, Tanoglu A, et al. Clinical usefulness of mean platelet volume and red blood cell distribution width to platelet ratio for predicting the severity of hepatic fibrosis in chronic hepatitis $B$ virus patients. Eur J Gastroenterol Hepatol 2014;26:1320-4.

7. Lou Y, Wang M, Mao W. Clinical usefulness of measuring red blood cell distribution width in patients with hepatitis B. PLoS ONE 2012;7: e37644.

8. Liu $\mathrm{H}$, Zhang $\mathrm{H}$, Wan $\mathrm{G}$, et al. Neutrophil-lymphocyte ratio: a nove predictor for short-term prognosis in acute-on-chronic hepatitis $B$ liver failure. J Viral Hepat 2014;21:499-507.

9. Roxburgh CS, McMillan DC. Role of systemic inflammatory response in predicting survival in patients with primary operable cancer. Future Oncol 2010;6:149-63.

10. Miyake $\mathrm{Y}$, Iwasaki $\mathrm{Y}$, Terada R, et al. Systemic inflammatory response syndrome strongly affects the prognosis of patients with fulminant hepatitis. B J Gastroenterol 2007;42:485-92.

11. Lee SD, Kim SH, Kim YK, et al. Prognostic significance of preoperative peripheral blood monocyte ratio in patients with hepatocellular carcinoma. World J Surg 2014;38:2377-85.

12. Lin GN, Peng JW, Liu DY, et al. Increased lymphocyte to monocyte ratio is associated with better prognosis in patients with newly diagnosed metastatic nasopharyngeal carcinoma receiving chemotherapy. Tumour Biol 2014;35:10849-54.

13. Paik KY, Lee IK, Lee YS, et al. Clinical implications of systemic inflammatory response markers as independent prognostic factors in colorectal cancer patients. Cancer Res Treat 2014;46:65-73.

14. Stotz M, Szkandera J, Stojakovic T, et al. The lymphocyte to monocyte ratio in peripheral blood represents a novel prognostic marker in patients with pancreatic cancer. Clin Chem Lab Med 2015:53:499-506.

15. Belotti A, Doni E, Bolis S, et al. Peripheral blood lymphocyte/ monocyte ratio predicts outcome in follicular lymphoma and in diffuse large B-cell lymphoma patients in the rituximab era. Clin Lymphoma Myeloma Leukoc 2015;15:208-13.

16. Merekoulias G, Alexopoulos EC, Belezos T, et al. Lymphocyte to monocyte ratio as a screening tool for influenza. PLoS Curr 2010;2: RRN1154.

17. Chinese Society of Infectious Diseases and Parasitic Diseases, Chinese Medical Association. Management scheme of diagnostic and therapy criteria of viral hepatitis. Chin $J$ Hepatol 2000;8:324-9.

18. Chinese Society of Hepatology and Chinese Society of Infectious Diseases-Chinese Medical Association. [The guideline of prevention and treatment for chronic hepatitis B (2010 version)]. ZhonghuaGan Zang Bing Za Zhi 2011;9:13-24.

19. Harrison PM. Management of patients with decompensated cirrhosis. Clin Med 2015;15:201-3.

20. Chung GE, Lee JH, Kim YJ. Does antiviral therapy reduce complications of cirrhosis? World J Gastroenterol 2014;20:7306-11.

21. Kamath PS, Wiesner RH, Malinchoc M, et al. A model to predict survival in patients with end-stage liver disease. Hepatology 2001;33:464-70. 
22. Tandon P, Garcia-Tsao G. Bacterial infections, sepsis, and multiorgan failure in cirrhosis. Semin Liver Dis 2008;28:26-42.

23. Antoniades CG, Wendon J, Vergani D. Paralysed monocytes in acute on chronic liver disease. J Hepatol 2005;42:163-5.

24. Mohammed NA, Abd El-Aleem S, Appleton I, et al. Expression of nitric oxide synthase isoforms in human liver cirrhosis. $J$ Pathol 2003;200:647-55. doi:10.1002/path.1377

25. Li Y, Sun DY. The expression of HLA-DR and TLR4 of peripheral blood monocytes in patients with posthepatitis liver cirrhosis and clinical significance. Master degree thesis of Hebei medical university. http://d.g.wanfangdata.com.cn/Thesis Y2106080.aspx.

26. Lin CY, Tsai IF, Ho YP, et al. Endotoxemia contributes to the immune paralysis in patients with cirrhosis. $J$ Hepatol 2007:46:816-26.

27. Berry PA, Antoniades CG, Carey I, et al. Severity of the compensatory anti-inflammatory response determined by monocyte HLA-DR expression may assist outcome prediction in cirrhosis. Intensive Care Med 2011;37:453-60.

28. Berres ML, Schnyder B, Yagmur E, et al. Longitudinal monocyte human leukocyte antigen-DR expression is a prognostic marker in critically ill patients with decompensated liver cirrhosis. Liver Int 2009;29:536-43.

29. Chen TA, Tsao YC, Chen A, et al. Effect of intravenous albumin on endotoxin removal, cytokines, and nitric oxide production in patients with cirrhosis and spontaneous bacterial peritonitis. Scand $J$ Gastroenterol 2009;44:619-25.

30. Leithead JA, Rajoriya N, Gunson BK, et al. Neutrophil-to-lymphocyte ratio predicts mortality in patients listed for liver transplantation. Liver Int 2015;35:502-9.

31. Lombardo L, Capaldi A, Poccardi G, et al. Peripheral blood CD3 and CD4 T-lymphocyte reduction correlates with severity of liver cirrhosis. Int J Clin Lab Res 1995;25:153-6.

32. Naranbhai $\mathrm{V}, \mathrm{Kim} \mathrm{S}$, Fletcher $\mathrm{H}$, et al. The association between the ratio of monocytes:lymphocytes at age 3 months and risk of tuberculosis (TB) in the first two years of life. BMC Med 2014;12:120.

33. Gary T, Pichler M, Belaj K, et al. Lymphocyte-to-monocyte ratio: a novel marker for critical limb ischemia in PAOD patients. Int $J$ Clin Pract 2014;68:1483-7.

34. Biyik M, Ucar R, Solak Y, et al. Blood neutrophil-to-lymphocyte ratio independently predicts survival in patients with liver cirrhosis. Eur J Gastroenterol Hepatol 2013;25:435-41. 